\title{
Demographic Profile of Pediatric Malignancies in Himachal Pradesh
}

\begin{abstract}
Context: Pediatric malignancy is on the rise worldwide, and it is imperative to know the profile of pediatric malignancy in different geographical areas so as to formulate effective strategy to its control. As there are no data from this part, especially the Himalayan region of North India, the present study was planned. Aims: The aims of this study were to study the pattern and demographic profile of pediatric malignancies at a tertiary care teaching hospital in Himachal Pradesh. Settings and Design: This was a hospital-based, prospective, nonrandomized, observational study. Subjects and Methods: Pattern and demographic profile of clinically suspected malignancies in children and adolescents (0-18 years) over a period of 1 year from June 2012 to May 2013 in the Department of Pediatrics and Radiotherapy. Statistical Analysis Used: Data were analyzed statistically using Epi Info software for all ratios and proportions. Results: A total of 31 children were diagnosed to have malignancy, which accounted for $0.86 \%$ of total admissions with a male-to-female ratio of 1.58:1.The three most common childhood malignancies were leukemia $(29.03 \%)$, central nervous system (CNS) tumors $(25.80 \%)$, and lymphoma $(19.35 \%)$, and these were followed by Wilm's tumor and hepatoblastoma, each accounting for $06.45 \%$ of total childhood malignancies. Hematological malignancies accounted for $48.38 \%$ and nonhematological malignancies for $51.62 \%$ of all the malignancies. Acute lymphoblastic leukemia was the most common hematological malignancy noted in $22.58 \%$, followed equally by Hodgkin's disease and non-Hodgkin's lymphoma $(20 \%$ of total hematological malignancies). Among nonhematological malignancies, CNS malignancies were the most common. In the age group of $0-5$ years, all cases of neuroblastoma, yolk sac tumor, and ovarian tumor and $50 \%$ cases of hepatoblastoma and Wilm's tumor were seen. In the age group of 5-10 years, leukemia (44.44\%) was the most common malignancy followed by lymphoma (33.33\%). In 14-18 years, CNS malignancies (50\%) were the most common malignancies followed by lymphoma $(33.33 \%)$. Conclusions: Hematological malignancies are the most common malignancies in the pediatric age group, and acute leukemia was the single most common pediatric malignancy, and CNS malignancies were the most common nonhematological malignancies.

Keywords: Himalayas, malignancies, North India, pediatric
\end{abstract}

\section{Introduction}

In 2008, 7.6 million people died of cancer $-13 \%$ of all deaths worldwide. About $70 \%$ of all cancer deaths occur in low- and middle-income countries. ${ }^{[1]}$ Worldwide, the annual number of new cases of childhood cancer exceeds 200,000 and more than $80 \%$ of these are from the developing world. ${ }^{[2]}$ Cancer remains one of the major causes of death in children between the ages of $0-14$ years. ${ }^{[3]}$ Pediatric cancers differ markedly from adult cancers in their nature, distribution, and prognosis. The incidence of childhood cancer and type vary greatly throughout the world. Although lower compared with the incidence of some adult cancers, it comes next to accidents as the leading cause of death among children

This is an open access journal, and articles are distributed under the terms of the Creative Commons Attribution-Non Commercial-ShareAlike 4.0 License, which allows others to remix, tweak, and build upon the work non-commercially, as long as appropriate credit is given and the new creations are licensed under the identical terms.

For reprints contact: reprints@medknow.com in the developed world, accounting for $10 \%-12.3 \%$ of all childhood deaths. ${ }^{[3-6]}$ In 2005, the International Union Against Cancer reported that, worldwide, more than 160,000 children are diagnosed with cancer per year, and about 90,000 die from cancer because of late presentation due to parental ignorance and poverty and poor health facilities. ${ }^{[7]}$ It has also been estimated that more than $85 \%$ of childhood cancer cases occurred in resource-poor countries, and it is possible that this will increase to $90 \%$ in the next two decades as a result of an expected rapid increase in the youth population. ${ }^{[8]}$ In developing countries like India, childhood mortality is still mainly due to malnutrition and infections, but pediatric tumors are also rising in number. ${ }^{[4]}$ As there is paucity of studies regarding the pattern of childhood malignacies from this part of north India, present study

\footnotetext{
How to cite this article: Badhan A, Bhardwaj P, Yadav V, Grover N, Seem RK. Demographic profile of pediatric malignancies in Himachal Pradesh. Indian J Med Paediatr Oncol 2018;39:287-91.
}

\section{Anupam Badhan, Parveen Bhardwaj, Vijay Yadav, Neelam Grover, Rajeev Kumar Seem ${ }^{1}$}

Departments of Pediatrics and ${ }^{1}$ Radiotherpy, Indira Gandhi Medical College, Shimla, Himachal Pradesh, India

Address for correspondence: Dr. Parveen Bhardwaj, Department of Pediatrics, Indira Gandhi Medical College, Shimla, Himachal Pradesh, India. E-mail: parveenbhardwaj@ hotmail.com 
was conducted to ascertain the pattern of childhood malignancies in Himachal Pradesh.

\section{Subjects and Methods}

A prospective nonrandomized study was conducted in children (0-18 years) over a period of 1 year from June 2012 to May 2013 in the Department of Pediatrics and Radiotherapy at a tertiary teaching institute in North India. All the children aged $\leq 18$ years with malignancies diagnosed by means of cytological or histopathological examination were enrolled in the study. Written informed consent was obtained from the parents/guardians of all patients. The study was approved by the Institutional Ethics Committee. The profile of childhood cancer was studied focusing on the proportion of malignancies according to (a) age at presentation (divided into four groups, i.e., 0-4 years, 5-9 years, 10-14 years, and 15-18 years), (b) sex, (c) rural and urban distribution, (d) site of origin of tumor, and (e) their histopathological diagnosis.

The cases were assessed clinically by taking relevant history and doing general physical examination. Specific investigations were undertaken to establish the diagnosis of malignancy. Hemoglobin, total leukocyte count, differential leukocyte count, and platelets count were analyzed by "COULTER'S MX-9" autoanalyzer. Bone marrow films were prepared from aspirated material and were stained using Romanowsky stain and examined under microscope. FNAC material obtained was smeared on glass slides fixed in 95\% ethyl alcohol for Papanicolaou staining and air dried for Giemsa staining. Excision, incision, and true cut biopsy were done where ever indicated. Specialized stains were used for more accurate diagnoses. Radiological investigations such as X-ray, ultrasound, computed tomography (CT) scans, and magnetic resonance imaging (MRI) were done. Data were analyzed statistically using Epi Info software for all ratios and proportions.

\section{Results}

In this study period, out of 3605 children admitted, 31 were diagnosed to have malignancy, which accounted for $0.86 \%$ of admission, and a total of 1978 patients (children + adult) were diagnosed to have one or the other malignancy during this period; thus, childhood malignancies $(n=31)$ constituted $1.57 \%$ of total cancer burden. There were 19 males and 12 females. Males were affected (61.29\%) more than females $(38.71 \%)$ with a male-to-female ratio of $1.58: 1$.

In this study, the three most common childhood malignancies were leukemia (29.03\%), central nervous system (CNS) tumors (25.80\%), and lymphoma (19.35\%) these were followed by Wilm's tumor and hepatoblastoma, each accounting for $06.45 \%$ of total childhood malignancies as shown in Table 1.

\begin{tabular}{lll}
\hline \multicolumn{2}{c}{ Table 1: Types of malignancies diagnosed } \\
\hline Site & Malignancy & Number of cases \\
\hline $\begin{array}{l}\text { 1) Hematological } \\
\text { 1a) Leukemia }\end{array}$ & \\
1aa) ALL & ALL - L1 & 3 \\
\multicolumn{1}{l}{ 1ab) AML } & ALL - L2 & 4 \\
& AML - M2 & 1 \\
1b) Lymphoma & AML -M3 & 1 \\
1ba) NHL & & \\
1bb) HL & HL-(MC) & 3 \\
& HL-(LP) & 2 \\
2) Nonhematological & & 1 \\
2a) CNS & Craniopharyngioma & 3 \\
& Medulloblastoma & 2 \\
& Meningioma & 1 \\
& Pinealoblastoma & 1 \\
2b) Neuroendocrine & Thalamic glioma & 1 \\
2c) Thorax & Teraroblastoma & 1 \\
2d) Hepatobiliary & mediastinum & 1 \\
2e) Renal & Hepatoblastoma & 2 \\
2f) Others & Wilm's tumor & 2 \\
All malignancies & Yolk sac tumor & 1 \\
\hline ALL - Acurian tumor & \\
\hline
\end{tabular}

$\overline{\text { ALL - Acute lymphoblastic leukemia; AML - Acute myeloid }}$ leukemia; NHL - Non-Hodgkin lymphoma; HL - Hodgkin lymphoma; CNS - Central nervous system; MC - Mixed cellularity; LP - Lymphocyte predominant

Hematological malignancies accounted for $48.38 \%$ and nonhematological malignancies for $51.62 \%$ of total pediatric malignancies.

In hematological malignancies, acute lymphoblastic leukemia was the most common accounting for $22.58 \%$ of total malignancies, $39.53 \%$ of total hematological malignancies, and $77.78 \%$ of acute leukemia. Among hematological malignancies, acute lymphoblastic leukemia is followed equally by Hodgkin's disease and non-Hodgkin's lymphoma (20\% of total hematological malignancies each) followed by acute myeloid leukemia (13.33\% of total hematological malignancies).

Among nonhematological malignancies, CNS malignancies accounted for $50 \%$ of malignancies followed by Wilm's tumor and hepatoblastoma, each accounting for $06.45 \%$ of total childhood malignancies and $12.5 \%$ of total nonhematological malignancies. These were followed by sympathetic nervous system tumors (neuroblastoma), anterior mediastinal teratoma, yolk sac tumor, and ovarian tumor each accounting for $3.23 \%$ of total childhood malignancies and $6.25 \%$ of total nonhematological malignancies.

Craniopharyngioma was the most common CNS malignancy. It accounted for $9.68 \%$ of total malignancies, 
Table 2: Age distribution of different childhood malignancies

\begin{tabular}{|c|c|c|c|c|c|c|}
\hline \multirow{2}{*}{$\begin{array}{l}\text { Serial } \\
\text { number }\end{array}$} & \multirow[t]{2}{*}{ Malignancy } & \multicolumn{5}{|c|}{ Age group } \\
\hline & & $0-5$ years $(\%)$ & $5-10$ years $(\%)$ & $10-14$ years $(\%)$ & $14-18$ years $(\%)$ & Total $<18$ years $(100 \%)$ \\
\hline 1 & Hematological & $3(20.00)$ & $7(46.67)$ & $3(20.00)$ & $2(13.33)$ & 15 \\
\hline $\mathrm{a}$ & Leukemias & $2(22.22)$ & $4(44.45)$ & $1(11.11)$ & $2(22.22)$ & 9 \\
\hline $\mathrm{b}$ & Lymphomas & $1(16.67)$ & $3(50.00)$ & $2(33.33)$ & 0 & 6 \\
\hline 2 & CNS malignancy & $2(25.00)$ & $1(12.50)$ & $2(25.00)$ & $3(37.50)$ & 8 \\
\hline 3 & Neuroblastoma & $1(100)$ & 0 & 0 & 0 & 1 \\
\hline 4 & Teratoma anterior mediastinum & 0 & 0 & 0 & $1(100)$ & 1 \\
\hline 5 & Hepatoblastoma & $1(50)$ & 0 & $1(50)$ & 0 & 2 \\
\hline 6 & Wilm’s tumor & $1(50)$ & $1(50)$ & 0 & 0 & 2 \\
\hline 7 & Yolk sac tumor & $1(100)$ & 0 & 0 & 0 & 1 \\
\hline 8 & Ovarian tumor & $1(100)$ & 0 & 0 & 0 & 1 \\
\hline Total & All malignancies & $10(32.26)$ & $09(29.04)$ & $6(19.35)$ & $6(19.35)$ & 31 \\
\hline
\end{tabular}

CNS - Central nervous system

$18.75 \%$ of total nonhematological malignancies, and $37.5 \%$ of CNS malignancies. Medulloblastoma was the second most common CNS malignancy. It accounted for $25 \%$ of total CNS malignancies followed by meningioma, pinealoblastoma, and thalamic glioma accounting for $12.5 \%$ of total CNS malignancies each as shown in Table 1.

Table 2 shows the age-wise distribution of pediatric malignancies.

In the age group of $0-5$ years, nonhematological malignancies were more common $(70.00 \%)$ than the hematological malignancies (30.00\%). Leukemia (20.00\%) was the most common malignancy in the $0-5$-year group. All cases of leukemia were ALL. CNS malignancies were the most common nonhematological malignancies. All cases of neuroblastoma, yolk sac tumor, and ovarian tumor were in this age group. Almost $50 \%$ cases of hepatoblastoma and Wilm's tumor were in this age group. In the age group of 5-10 years, hematological malignancies were more common $(77.78 \%)$ than the nonhematological malignancies $(22.22 \%)$. Leukemia $(44.44 \%)$ was the most common malignancy followed by lymphoma (33.33\%). Nearly $75 \%$ cases of leukemia were ALL and $25 \%$ were AML. Only two cases of nonhematological malignancies were as follows: one of craniopharyngioma and one of Wilm's tumor. Almost 50\% cases of Wilm's tumor were in this age group. In the age group of 10-14 years, hematological malignancies $(n=3)$ and nonhematological malignancies $(n=3)$ were equally common. Lymphoma $(33.33 \%)$ was the most common malignancy in this age group. Among hematological malignancies, lymphoma (66.67\%) was more common than leukemia (33.33\%). All cases of leukemia were ALL. CNS (66.67\%) malignancies were the most common nonhematological malignancies. In the age group of 14-18 years, hepatoblastoma constituted $16.67 \%$ of cases in this age group. Almost 50\% cases of hepatoblastoma were in this age group. Nonhematological malignancies were more common $(66.67 \%)$ than the hematological malignancies $(33.33 \%)$. CNS malignancies $(50 \%)$ were the

\begin{tabular}{llc}
\hline \multicolumn{2}{c}{ Table 3: Percentage of pediatric malignancies } \\
\hline Study & Place & $\begin{array}{c}\text { Burden (percentage } \\
\text { of total malignancies) }\end{array}$ \\
\hline Rathi et al..$^{[4]}$ & New Delhi & 3.38 \\
Yeole, et al. ${ }^{[1]}$ & Mumbai & 3.3 \\
Jignasa and Mandakini ${ }^{[10]}$ & Surat(Gujarat) & 2.0 \\
Ferlay et al. ${ }^{[12]}$ & USA & 0.8 \\
Arora et al..$^{[2]}$ & UK & 0.5 \\
Present study & HP & 1.57 \\
\hline
\end{tabular}

most common malignancies in this age group followed by lymphoma (33.33\%). Craniopharyngioma, meningioma, and pinealoblastoma were equally common. Lymphoma was more common (66.67\%) than leukemia (33.33\%). ALL and AML were equally common. All cases of teratoma were in this age group.

\section{Discussion}

A limited number of retrospective and prospective studies have been conducted on demographic profile of childhood malignancies all over the world and more so from India and especially from the Northern part of the country. Most of these studies are on hematological malignancies, especially acute leukemia, the most common malignancy being observed all over the world among children. There are a very few places, especially in the western countries, ${ }^{[9]}$ where cancer registration system has been established and well maintained and that is why population-based studies can be conducted in those countries. Since our country lacks a well-maintained cancer registration system, we have to rely on hospital-based studies for epidemiological as well as other statistical data. In this study, the pediatric tumors constituted $1.57 \%$ of all the malignancies; our result is similar to Jignasa and Mandakini[ ${ }^{[10]}$ where they found $2.0 \%$ childhood cancer among all malignancies. Table 3 shows the comparison of various studies.

In this study, nonhematological malignancies were more common $(51.62 \%)$ than the hematological malignancies $(48.38 \%)$ and the three most common 


\begin{tabular}{|c|c|c|c|c|c|c|c|c|}
\hline Tumor & USA $^{[13]}$ & $\mathbf{U K}^{[14]}$ & Australia[15] & Indig $[4]$ & Korolo[5] & Dolhi[16] & & \\
\hline & & & & & & & & \\
\hline Leukemia & 30.1 & 20.4 & 20.6 & 32.00 & 30.0 & 28.8 & 32.80 & 29.03 \\
\hline Lymphoma & 12.3 & 8.7 & 10.3 & 14.08 & 10.0 & 11.5 & 12.65 & 19.35 \\
\hline CNS tumors & 19.1 & 16.6 & 21.0 & 18.21 & 19.3 & 21.0 & 17.60 & 25.80 \\
\hline Neuroblastoma & 8.1 & 7.5 & 7.4 & 4.21 & 5.1 & 4.1 & - & 03.23 \\
\hline Wilm’s tumor & 6.5 & 5.4 & 6.6 & 4.94 & 5.4 & 3.3 & 5.25 & 06.45 \\
\hline Bone tumors & 4.8 & 4.8 & 5.3 & 7.66 & 5.4 & 3.3 & 3.85 & 0 \\
\hline Soft-tissue tumors & 6.3 & 8.5 & - & 5.49 & 6.6 & 3.8 & 4.30 & 0 \\
\hline Other & 10.1 & 16.0 & 17.1 & 18.35 & 13.8 & 11.8 & 19.00 & 16.13 \\
\hline
\end{tabular}

CNS - Central nervous system

childhood malignancies were leukemia (29.03\%), CNS tumors $(25.80 \%)$, and lymphoma (19.35\%). It is comparable with the data from the developed world, for example, in the USA, ${ }^{[10]}$ the three most frequent childhood cancers diagnosed were leukemia $(30.2 \%)$, CNS cancers $(21.7 \%)$, and lymphomas (10.9\%).

Leukemias were the most common form of childhood malignancies and together with lymphoma constituted $30 \%-50 \%$ of all malignancies in different regions as seen in Table 4. In the present study, lymphoma and leukemia together constituted $48.38 \%$ of childhood malignancies. Among leukemias, the most common in different studies were acute leukemias, $75 \%-80 \%$ being ALL and $20 \%-25 \%$ AML. ${ }^{[11]}$ In the present study, ALL constitutes $77.78 \%$ of total leukemias and AML was $22.22 \%$ of leukemias.

In the present study, the incidence of pediatric CNS tumor is $25.80 \%$, which is almost comparable with a study which is carried out at Delhi, ${ }^{[15]}$ but the incidence is much higher in our study than other studies done in and outside India. This may be because of round-the-clock availability of high-quality CT and MRI and early imaging of pediatric patients done on priority basis in our hospital. The incidence of Wilm's tumor $(06.45 \%)$ is almost comparable with the data from the studies conducted in developed countries such as the USA, ${ }^{[13]}$ Australia, ${ }^{[15]}$ and developing African countries ${ }^{[14]}$ but slightly higher than other Indian studies. ${ }^{[4,5,16]}$ The proportion of neuroblastoma $(03.23 \%)$ in our study was much lower than studies conducted in developed countries such as the USA, ${ }^{[13]}$ the UK, ${ }^{[14]}$ and Australia ${ }^{[15]}$ but comparable with different Indian studies. ${ }^{[4,5,16]}$ No case of bone tumors, soft-tissue tumors were diagnosed during this study period of 1 year. The incidence of childhood malignancies is higher in male than in female. Male and female ratio in the present study is $1.58: 1$, which is almost quite similar to Yeole et al ${ }^{[11]}$ and Joshi and Kumar. ${ }^{[17]}$

Male preponderance in our country could also be as a result of our cultural factors wherein boys get more attention and are brought to the hospital more often for management. ${ }^{[10]}$ The highest incidence was seen in $0-5$ years $(32.26 \%)$, followed by $5-10$ years $(29.04 \%), 10-14$ years $(19.35 \%)$, and 14-18 years $(19.35 \%)$. The incidence of childhood malignancies varied among different age groups. Although except this study, rest of the studies have not included adolescents above 14 years, so proportions in different age groups cannot be compared, but trends in our study are almost similar to Yeole et al..$^{[1]}$ This study shows that the overall incidence of childhood malignancies was more in 0-5 years of age group and decreases gradually with age toward late adolescence and this is in concordance with other Indian studies. ${ }^{[10,11]}$ The number of cases belonging to rural area $(90.32 \%)$ is more than those belonging to an urban area $(9.68 \%)$ which is well comparable with the general population.

\section{Conclusions}

In this part of the country, the three most common malignancies are leukemia (29.03\%), CNS tumors $(25.80 \%)$, and lymphoma (19.35\%). Nonhematological malignancies are more common $(51.62 \%)$ than the hematological malignancies (48.38\%). Acute lymphoblastic leukemia is the most common hematological malignancy. The most common nonhematological malignancies are CNS malignancies followed by Wilm's tumor and hepatoblastoma. Craniopharyngioma is most common CNS malignancy. Acute leukemia is most common in 5-10 years' age group and CNS malignancies are most common in 14-18 years' age group (37.50\%). Male preponderance is seen in hematological malignancies $(86.67 \%)$ (both leukemia and lymphoma). Female preponderance is seen in nonhematological malignancies. The number of cases belonging to rural area $(90.32 \%)$ was more than those belonging to an urban area $(9.68 \%)$.

Financial support and sponsorship

Nil.

\section{Conflicts of interest}

There are no conflicts of interest.

\section{References}

1. WHO. 10 Facts about Cancer. Available from: http://www.who. int/features/factfiles/cancer/facts/en/index9.html. [Last accessed on 2012 May 03].

2. Arora RS, Eden TO, Kapoor G. Epidemiology of childhood cancer in India. Indian J Cancer 2009;46:264-73. 
3. American Cancer Society. Cancer Facts and Figures; 2005. Available from: http://www.cancer.org. [Last reviewed on 2005 Mar 29].

4. Rathi AK, Kumar S, Ashu A, Singh K, Bahadur AK. Epidemiology of paediatric tumors at a tertiary care centre. Indian J Med Paediatr Oncol 2007;28:343-50.

5. Kusumakumary P, Jacob R, Jothirmayi R, Nair MK. Profile of pediatric malignancies: A ten year study. Indian Pediatr 2000;37:1234-8.

6. Kusumakumari P. Childhood malignancies. Indian J Med Paediatr Oncol 1996;17:168-77.

7. International Union against Cancer. The "My Child Matters" Initiative [press release]. Geneva: UICC; 2005. Available from: http://www.uicc.org/index.php?option=com_content \&task $=$ view\&id $=15898 \&$ Itemid $=43$. [Last accessed on 2008 Nov 12].

8. Yaris N, Mandiracioglu A, Büyükpamukcu M. Childhood cancer in developing countries. Pediatr Hematol Oncol 2004;21:237-53.

9. Mandong BM, Angyo IA, Zoakah AI. Paediatric solid malignant tumours in JUTH, Jos (hospital based histopathology study).
Niger J Med 2000;9:52-5.

10. Jignasa B, Mandakini P. Profile of pediatric malignancy: A three year study. Natl J Community Med 2011;2:24-7.

11. Yeole BB, Advani SH, Sunny L. Epidemiological features of childhood cancers in greater Mumbai. Indian Pediatr 2001;38:1270-7.

12. Ferlay J, Bray F, Pisani P, Parkin DM. GLOBOCAN 2002: Cancer incidence, mortality and prevalence worldwide. Lyon: IARC Press; 2004.

13. Young JL Jr., Miller RW. Incidence of malignant tumors in U. S. Children. J Pediatr 1975;86:254-8.

14. Pearson D, Steward JK. Malignant disease in juveniles. Proc R Soc Med 1969;62:685-8.

15. McWhirter WR, Siskind V. Childhood cancer survival trends in Queensland 1956-80. Br J Cancer 1984;49:513-9.

16. Arya LS. Childhood cancer - Challenges and opportunities. Indian J Pediatr 2003;70:159-62.

17. Joshi V, Kumar A. Pediatric Hemato-Oncology in India. Epidemiologic Differences. M.B. Agrawal. Hematology Today; 2004. 